\title{
Noninvasive Micrometer-Scale Particle-Beam Size Measurement Using Optical Diffraction Radiation in the Ultraviolet Wavelength Range
}

\author{
M. Bergamaschi®, ${ }^{1,2, *}$ A. Aryshev $\odot,{ }^{3}$ P. Karataev, ${ }^{2}$ R. Kieffer® ${ }^{1,2}$ T. Lefevre $\odot,{ }^{1}$ S. Mazzoni $\odot,{ }^{1}$ \\ N. Terunuma, ${ }^{3}$ and R. Yang ${ }^{4, \dagger}$ \\ ${ }^{1}$ CERN, CH-1211 Geneva 23, Switzerland \\ ${ }^{2}$ John Adams Institute at Royal Holloway, University of London, Egham, Surrey TW20 OEX, United Kingdom \\ ${ }^{3}$ KEK: High Energy Accelerator Research Organization, 1-1 Oho, Tsukuba, Ibaraki 305-0801, Japan \\ ${ }^{4}$ LAL, Universite Paris-Sud, CNRS/IN2P3, Orsay, France
}

(Received 1 July 2019; revised manuscript received 15 November 2019; published 23 January 2020)

\begin{abstract}
We present recent achievements in the application of optical diffraction radiation (ODR) to the measurement of the transverse beam size of a $1.3 \mathrm{GeV}$ micrometer-size electron beam performed with an instrument installed in the extended extraction line of the KEK Accelerator Test Facility. ODR is a recent technique for the measurement of the transverse size and emittance of highly relativistic particle beams. ODR has the advantage of being a noninvasive and relatively inexpensive technique and is a candidate for the operation of linear particle accelerators, where no simple alternatives (e.g., synchrotron radiation) are available. In an effort to improve the resolution and performance, we establish an alternative target microfabrication technology, adopt solutions to improve the signal-to-noise ratio, and perform an experiment in the UV spectrum at $250 \mathrm{~nm}$. With such a configuration, a transverse beam size as low as $3 \mu \mathrm{m}$ is measured, drastically improving on past measurements reported in the literature. In light of these results, ODR, when combined with a high-resolution optical-transition-radiation monitor, represents a credible diagnostics solution for low-emittance high-intensity particle beams.
\end{abstract}

DOI: 10.1103/PhysRevApplied.13.014041

\section{INTRODUCTION}

In particle accelerators, the measurement of the particle beam's transverse profile is a key diagnostic for the assessment of the beam emittance and for safe operation of the machine [1,2]. Since the early years of operation of accelerators, several methods and techniques for measurement of the transverse beam profile have been developed and tested. These include the insertion of metallic wires or grids into the beam path to generate secondary particles (e.g., photons or electrons) [3], the injection of gas into the beam path volume to generate ionization or fluorescence $[4,5]$, and the analysis of synchrotron radiation (SR) from bending magnets or undulators [6]. Here we restrict our attention to a method, perhaps one of the most widespread ones, that relies on the use of optical transition radiation (OTR), an effect discovered by Ginzburg and Frank

*michele.bergamaschi@cern.ch

†Present address: CERN, CH-1211 Geneva 23, Switzerland.

Published by the American Physical Society under the terms of the Creative Commons Attribution 4.0 International license. Further distribution of this work must maintain attribution to the author(s) and the published article's title, journal citation, and DOI. in $1945[7,8]$. OTR refers to the emission of broadband electromagnetic radiation when a near-relativistic charged particle crosses a boundary between media with different dielectric constants [8]. OTR is emitted through the fast polarization of the atoms of the transition medium (e.g., a metallic sheet in vacuum, often referred to as an "OTR screen") by the electromagnetic field associated with the moving particle. The popularity of OTR in the field of accelerator beam diagnostics is due to a number of advantages: a high light yield of approximately 1 photon per 100 particles; a simple and rather inexpensive experimental setup where the beam profile corresponds, in most cases, to that of an OTR source produced by a metallic screen inserted in the beam path; the possibility of working at different wavelengths; and the temporally "instantaneous" emission, which allows one to recover the temporal profile of the particle beam. OTR can still be used in the case of small micrometer-scale particle beams, where the beam size is measured from the visibility of the so-called point spread function (PSF) [9]. Notwithstanding these advantages, OTR is an invasive method; that is, the particle beam must cross a thin (e.g., a few hundred micrometers) metallic sheet inserted into the beam path. As such, OTR screens might perturb the beam and cause an emittance blowup and a shower of secondary particles due to the interaction 
with the screen material. Another case, very relevant in high-energy physics, where OTR screens cannot be used is in colliders for particle-physics experiments, where very intense beams are present to maximize the occurrence of collision events and minimize the residual background of the detector. In such a case, the energy deposition inside the OTR-screen material becomes so high that the melting temperature can be exceeded, causing irreversible damage to the screen [10]. For this reason, noninvasive techniques are required in colliders or in any accelerator with a very intense beam. The state of the art in noninvasive (i.e., techniques that do not perturbing the particle beam to be measured, or do so minimally) transverse-beam-profile and emittance diagnostics for ultrarelativistic electron and positron beams is based on the so-called laser-wire technique [11-13], where a thin laser beam is scanned across a particle beam and inverse-scattered photons are registered downstream. This technique requires an expensive laser, sophisticated final focus optics, and a team of experts to operate the system. We focus our attention on a simpler and cheaper alternative: optical diffraction radiation (ODR). ODR was proposed in 1997 by Castellano [14] as a beam size measurement technique. ODR can be considered as a noninvasive version of OTR, as it shares the same light-emitting physical process. In ODR, in fact, the particle beam passes through a narrow rectangular aperture in a metallic foil, instead of going through the foil, and therefore only a very minute fraction of the beam energy is dissipated in the target in the form of heat. This poses no limitation on the use of ODR as a technique in high-intensity colliders, where charge densities in excess of $10^{8} \mathrm{nC} / \mathrm{cm}^{2}$ are present [15]. In addition, ODR does not perturb the beam emittance. The method for detection of ODR is in principle simple and very similar to that for OTR, that is, an optical instrument that allows one to record the angular distribution of the ODR intensity with a camera. It is in fact possible to extract particle-beam parameters such as the beam size (i.e., beam emittance), the angular divergence, and the position at the slit location, knowing precisely the dimensions and the alignment of the slit; the effect of these parameters has been studied in detail by several authors $[14,16]$. The first attempt to apply this method to beam diagnostics was reported in Ref. [17], followed by several subsequent studies [18,19]. The best reported sensitivity to beam size $(14 \mu \mathrm{m})$ was achieved in the optical wavelength range, a very promising result for a noninvasive technique but nevertheless not sufficient for future linear colliders, where a resolution smaller than $10 \mu \mathrm{m}$ is required. Despite these advantages, ODR is still an "expert" technique and, to the knowledge of the authors, is not used in the operation of any collider or light source where either tolerance to high intensity or noninvasive techniques are needed. In the opinion of the authors, this is mainly due to some of the technical challenges associated with the fabrication of a target of optical quality with micrometer-size apertures, the need to reduce the SR background reflected by the target, and the complex data extraction.

In this paper we report a study of an alternative approach, investigated in the framework of linear-collider studies [20], that relies on the combined use of OTR for single-bunch operation (low charge), and on its noninvasive version, ODR, for full-beam operation. The OTR and ODR instrument is installed in the extraction line of the Accelerator Test Facility (ATF) at KEK (Tsukuba, Japan) for the measurement of micrometer-size beams. The low emittance of the ATF $(12-30 \mathrm{pm})$ at the exit of the damping ring makes the contribution of the angular divergence of the beam (approximately 2.6-6 $\mu \mathrm{rad}$ ) negligible in the ODR beam size measurements [18,21]. To ensure sufficient resolution, the optical instrument is designed to record radiation at different wavelengths, down to the UV range at $250 \mathrm{~nm}$. After preparatory experiments [22], a procedure for the fabrication of a suitable ODR target is established, with several prototypes produced at the École polytechnique fédérale de Lausanne (EPFL). Such a target consists of a series of rectangular micrometer-scale apertures on an optical-grade substrate that, when used after a mask, allow one to reduce significantly the SR background. Optical simulations derived from previous OTR studies by our group [23,24] are performed to confirm analytical beamsize predictions with ODR data [18]. OTR measurements can be easily performed with the same instrument to crosscalibrate ODR data and provide complementary measurements for low-intensity beams. The paper summarizes all technical challenges met during the experimental work, the solutions found, and the main achievements demonstrating the performance of the instrument.

\section{INSTRUMENT}

\section{A. Overview}

The experimental system is installed in the ATF2 beam line [25], as shown in Fig. 1, behind two strong quadrupoles that focus the beam to a vertical size varying from a few hundreds of nanometers to tens of micrometers [21]. The instrument is designed to measure the far-field angular distribution of the ODR, as this carries the information about the beam size $[14,17]$. Figure 2 provides a schematic drawing of the optical instrument. We refer to the letters in the figure to describe it. The electron bunches $\left(e^{-}\right)$travel over a mask (a) before passing through the target slit (b). The distance between the mask and the target ("dist") is $123 \mathrm{~mm}$. Considering the wavelength $(\lambda)$ observed during the experiment, this distance represents a fraction of the radiation formation zone $\left(L_{f}=\right.$ $\left.\gamma^{2} \lambda / 2 \pi\right)$; in particular, dist $\approx 0.3 L_{f}$ for $\lambda=400 \mathrm{~nm}$ and dist $\approx 0.48 L_{f}$ for $\lambda=250 \mathrm{~nm}[18,26]$, meaning that interference between the diffraction radiation produced from the mask and from the target must be taken into account 


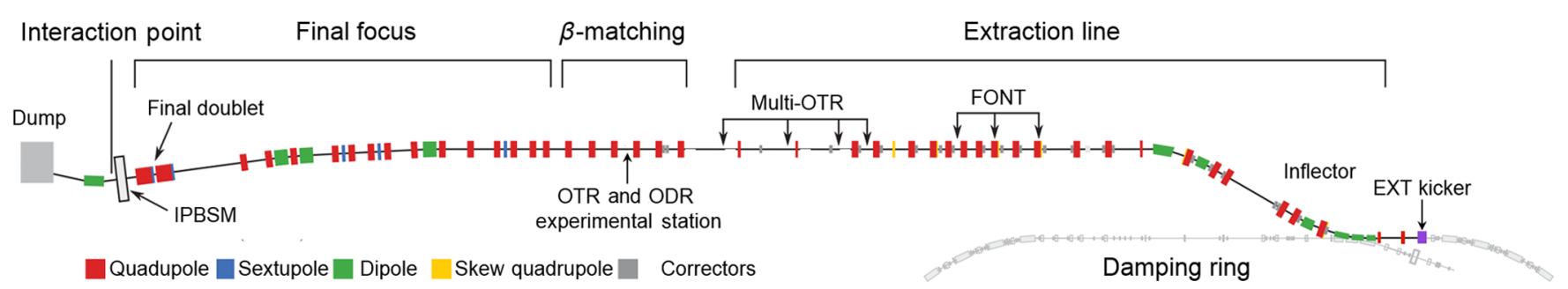

FIG. 1. Schematic layout of the ATF2 beam line: the location of the OTR and ODR experimental station in the matching section is shown. IPBSM: Interaction Point Beam Size monitor; FONT: Feedback On Nano-second Timescale; EXT: Extraction.

in the analysis, as shown in Ref. [18]. The far-field angular distribution of the radiation emitted by the ODR target (b) is measured by placing the imaging sensor in the back focal plane of a lens (g). The efficiency of such a measurement scheme was investigated experimentally in Ref. [27]. Furthermore, the setup is designed to allow both imaging of the target and recording of the angular distribution of the radiation to be done at the same time [28]. The imaging line (i) is used to center the target on the beam, and can be used as an optical beam position monitor (BPM), with the beam position deduced from the intensity imbalance between the two slit edges [29].

\section{B. Mechanical installation}

The instrument consists in a vacuum chamber installed on the main beam line, equipped with actuators to control the position of the target and mask (see Sec. IID), and UV-grade fused-silica viewports to send the radiation emitted by the target towards an optical instrument that records it in the visible and UV range. Figure 3(a) shows the instrument as installed in the extraction line

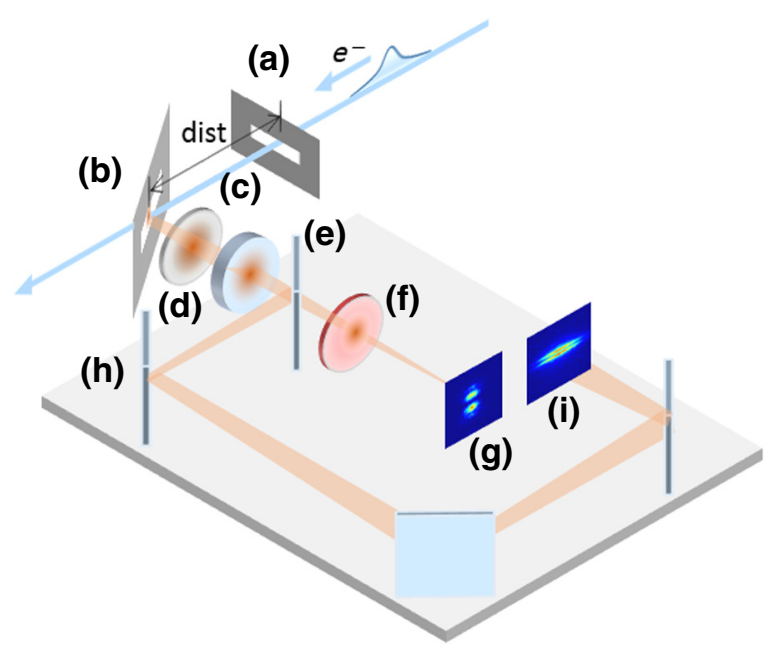

FIG. 2. Schematic drawing of the optical instrument: mask slit (a), target slit (b), polarizer (c), lens (d), optical beam splitter (e), mirrors (h), filter wheel (f). Both images (i) and the angular distribution $(\mathrm{g})$ of the ODR intensity of the electron bunches $e^{-}$ are recorded. of ATF2. The tank is connected to the ATF beam-line pipe through two DN40CF flanges [Fig. 3(b)]. Two largerdiameter flanges (DN63CF) on the side of the vacuum chamber are equipped with UV-grade fused-silica viewports that form angles of $40^{\circ}$ and $90^{\circ}$ with the beam axis. Two actuators are installed on dedicated flanges at the top of the vacuum chamber to control the vertical insertion of the ODR target and of a SR mask, as depicted in Fig. 4. In the case of the ODR target, the rotation and transverse position can also be controlled remotely.

On the opposite side of the vacuum chamber with respect to the two UV-grade fused-silica viewports, an actuator allows the horizontal insertion of an additional SR mask, and a second DN63CF flange is used to connect a NEXTorr D100-5 vacuum pump [30]. On the same side, there are two small viewports (DN16CF), tilted at angles of $45^{\circ}$ and $135^{\circ}$, that are used for optical-alignment purposes. When the ODR target and mask are not inserted into the beam line, a replacement chamber can be inserted from underneath between the DN40CF flanges to suppress the transverse wake field of the incoming beam, which can enlarge the beam size at the interaction point at the end of the ATF2 beam line. The replacement chamber is composed of an aluminum pipe of the same inner diameter as the main ATF2 beam pipe. Radio-frequency gaskets made of copper-beryllium alloy provide electrical contact with the main beam line. To obtain micrometer precision in the positioning of all components, the actuators are remotely controlled with stepper motors and encoders.

\section{Optical lines}

In this section, we refer to the letters in Fig. 2 to describe the optical line. The light is extracted through the $90^{\circ}$ viewport, passes through a motorized polarizer (UVT260A-25 from Moktek) (c) to select the desired polarization, and is split into two independent optical lines via an 8:92 opticalpellicle beam splitter (BP208 from Thorlabs) (e): one to create an image of the source, and the other to observe the angular distribution. A 2-inch-diameter planoconvex lens with a focal length of $250 \mathrm{~mm}$ (LA4538 from Thorlabs) (d) is installed before the beam splitter so that it is used in both optical lines. The lens is made of UV-grade fused silica without any coating, to allow light transmission from 
the far UV $(200 \mathrm{~nm})$ to the visible wavelength range up to $700 \mathrm{~nm}$. The imaging optical line consists of three 2inch UV-enhanced aluminum mirrors (PF20-03-F01 from Thorlabs) (h) that collect the light reflected by the beam splitter $(8 \%)$, to direct the radiation to a gated intensified CCD camera (dicam pro with a GaAs photocathode from PCO [31]) (i). The mirrors, suitable for a $250-700-\mathrm{nm}$ spectral range, are chosen to increase the distance between the lens and the camera so as to have the desired magnification on the CCD $(\times 1.8$ after demagnification due to the internal optical coupling of the intensifier CCD). In the angular-distribution optical line, the detection system is positioned directly in front of the beam splitter so that it can collect $92 \%$ of the radiation transmitted.
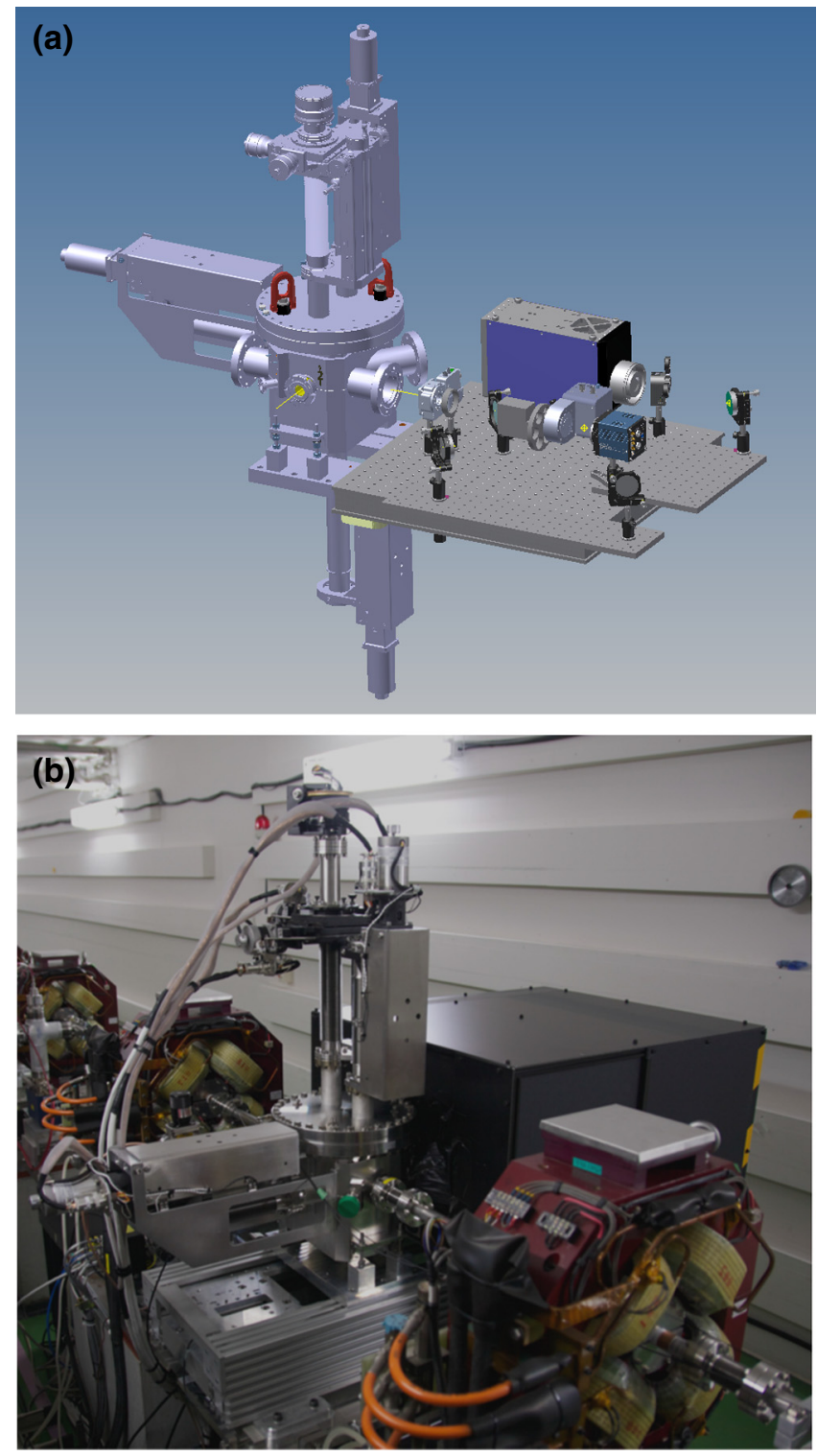

FIG. 3. Schematic illustration of the tank and optical instrument (a), and the tank and optical instrument installed in the extraction line of ATF2 (b).

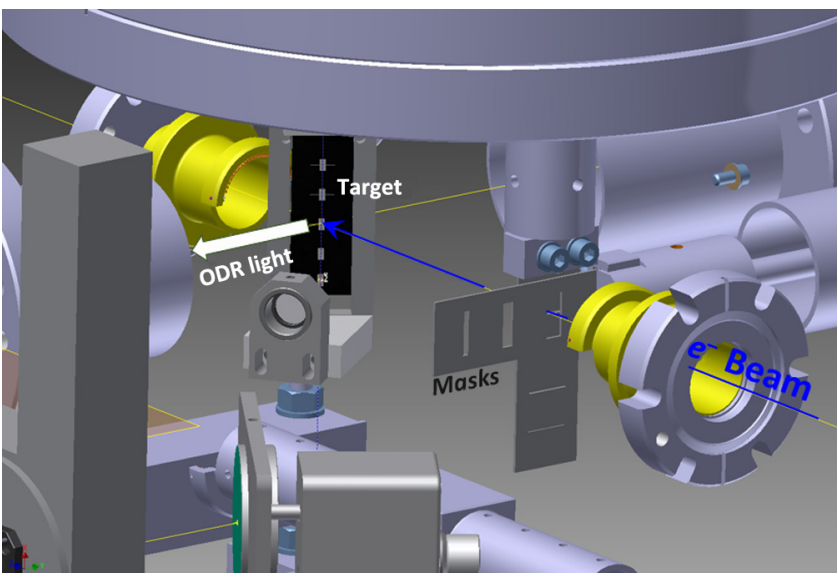

FIG. 4. In-vacuum components of the instruments. A set of two masks mounted on a linear actuator blocks most of the synchrotron radiation. The target generates transition and diffraction radiation in the direction of the optical instrument through a $90^{\circ}$ viewport.

A remotely controlled filter wheel (FRM 40-6-D25-HSM from OWIS [32]) (f) with a set of 1-inch band-pass filters is installed just before the detector to select different observation wavelengths and bandwidths. The filters used during data acquisition are the following: Thorlabs FB40010 (center peak wavelength $400 \mathrm{~nm}$, FWHM bandwidth $10 \mathrm{~nm}$ ), Thorlabs FB400-40 (center peak wavelength 400 nm, FWHM bandwidth $40 \mathrm{~nm}$ ), Thorlabs FB600-40 (center peak wavelength $600 \mathrm{~nm}$, FWHM bandwidth $40 \mathrm{~nm}$ ), eSource optics 25250FBB (center peak wavelength 250 $\mathrm{nm}, \mathrm{FWHM}$ bandwidth $40 \mathrm{~nm}$ ), and Andover 232FS10-25 (center peak wavelength $232 \mathrm{~nm}$, FWHM bandwidth 10 $\mathrm{nm})$. The detector is a gated image intensifier unit coupled to a scientific CMOS camera (g). The intensifier (C954704 from Hamamatsu) is a double-stage microchannel plate (MCP) with a multialkali photocathode sensitive down to 200-nm wavelength [33]. The camera (Edge 4.2LT from PCO [34]) is chosen for its very low readout noise (a mean value of 0.8 electrons). The camera and the intensifier, coupled by a relay lens (A11669 from Hamamatsu), are installed on a remotely controlled translation stage (TLSR150B-KT04 from Zaber Technologies) to scan across the direction of the incident light to find the back focal plane of the lens for the different observation wavelengths. Since the image-intensifier technology is based on a MCP, the cameras can be gated to a very short time window ( 20 ns for the PCO camera and 5 ns for the Hamamatsu intensifier). This allows us to reduce significantly the background noise and to increase the signal-to-noise ratio.

\section{Target specifications and fabrication steps}

In the ODR technique, the particle beam passes through a narrow rectangular slit, where radiation is produced by the interaction of the electromagnetic field of the particles 
with the slit edges. The light yield of ODR decays exponentially as a function of the slit width $d$ as $e^{-d / h}$, where

$$
h=\frac{\gamma \lambda}{2 \pi}
$$

is the radius of the effective field of the electron [14], $\lambda$ is the radiation wavelength, and $\gamma=1 / \sqrt{1-\left(v^{2} / c^{2}\right)}$ is the Lorentz factor. Any object placed within a distance $h$ from the particle trajectory will emit diffraction radiation. For typical ultrarelativistic beam parameters $(\gamma \propto 1000)$ and wavelengths in the UV-vis range (200 $\mathrm{nm} \leq \lambda \leq 700 \mathrm{~nm})$, the impact parameter falls in the range $30 \mu \mathrm{m} \leq h \leq$ $100 \mu \mathrm{m}$. To provide a sufficient light yield, the slit width $d$ must therefore be in the same range, while the slit must have a transverse length of a few millimeters to avoid perturbing the beam and to allow enough alignment tolerance. In addition, the coplanarity between the slit edges must be kept below a fraction of wavelength $(\leq \lambda / 10)$ to guarantee that the radiation produced by the two edges from the same particle sums coherently $[22,35]$. To allow measurement in the UV range down to $200 \mathrm{~nm}$, the coplanarity must therefore be better than $20 \mathrm{~nm}$. Given the stringent requirements, and after a few unsuccessful attempts at outsourcing the fabrication of the ODR target, we decided to develop our own fabrication process at the Centre of Micro-Nano Technology (CMi) at the EPFL. The procedure that leads to the best results is the outcome of a year-long exercise of establishing the correct sequence to fulfill the requirements. We describe here the procedure for the production of the ODR target. The same procedure (except for the selective depolishing of the surface - see Step 16) is applied to produce the SR mask, which has the same shape as the target but serves the purpose of blocking the SR produced by the dipoles and quadrupoles upstream.

The substrate used is a $1400-\mu \mathrm{m}$ thick monocrystalline silicon wafer of diameter $100 \mathrm{~mm}$ optically polished on both faces (surface roughness $0.3 \mathrm{~nm} \mathrm{rms}$ ). On this substrate, one ODR target and one mask, both with four slits, are produced with vertical sizes varying from a minimum of $49.7 \mu \mathrm{m}$ to a maximum of $399 \mu \mathrm{m}$, as shown in Fig. 5.

The process can conceptually be divided into three parts: (1) A crystal alignment procedure to obtain sharp slit edges, necessary for uniform ODR emission from the target. (2) Anisotropic etching of the slits in the silicon substrate, where the etch rates have to be taken into account in order to obtain the desired slit width on the opposite side of the silicon wafer. (3) Surface treatment, because a portion of the target is kept without apertures so as to have a flat highly reflective surface for OTR calibration (see Sec. III). Furthermore, a selective microsand-blasting treatment allows the background SR contribution during the ODR measurement to be reduced greatly. See Table I for an illustration of the fabrication steps.
TABLE I. Target fabrication steps. The $\mathrm{Si}_{3} \mathrm{~N}_{4}$ coating layer is shown in blue, the AZ9221 photoresist in red, the ProTEK protective layer in yellow, and the $\mathrm{MgF}_{2}$-protected aluminum coating in green.

\begin{tabular}{|c|c|c|c|}
\hline $\begin{array}{l}\text { Step. } \\
\text { no. }\end{array}$ & $\begin{array}{l}\text { Cross } \\
\text { section }\end{array}$ & $\begin{array}{l}\text { Step } \\
\text { no. }\end{array}$ & $\begin{array}{l}\text { Cross } \\
\text { section }\end{array}$ \\
\hline 1 & & 9 & \\
\hline 2 & & 10 & \\
\hline 3 & & 11 & \\
\hline 4 & & 12 & \\
\hline 5 & & 13 & \\
\hline 6 & & 14 & \\
\hline 7 & & 15 & \\
\hline 8 & & 16 & \\
\hline
\end{tabular}

\section{Crystal alignment procedure}

(a) Step 1: low-pressure chemical vapor deposition is performed in a furnace, allowing a $100-\mathrm{nm} \mathrm{Si}_{3} \mathrm{~N}_{4}$ coating layer to be built on the whole wafer (shown in blue).

(b) Step 2: a 2- $\mu \mathrm{m}$ photoresist layer (AZ9221, shown in red) is deposited by spin coating on the top.

(c) Step 3: a photolithographic mask is used to imprint a series of $60-\mu \mathrm{m}$-diameter circular dots on the wafer sides. After UV exposure, the resist is chemically developed.

(d) Step 4: plasma etching (chemistry based on $\mathrm{CHF}_{3}$ and $\mathrm{SF}_{6}$ ) of the unprotected $\mathrm{Si}_{3} \mathrm{~N}_{4}$ dielectric is performed with an Advanced Plasma System from SPTS Technologies.

(e) Step 5: after stripping the photoresist (shown in red), we perform silicon etching using potassium hydroxide $(\mathrm{KOH})\left(23 \%\right.$ and $\left.80^{\circ} \mathrm{C}\right)$ on a wet bench. In this step, we are now able to observe a small square for each dot due to anisotropic etching of the silicon by the $\mathrm{KOH}$. The tilt of the squares allows us to retrieve the crystal orientation to within a precision of $0.1^{\circ}$.

(f) Step 6: a 2- $\mu \mathrm{m}$ photoresist layer (AZ9221, shown in red) is again deposited by spin coating. 
(a)

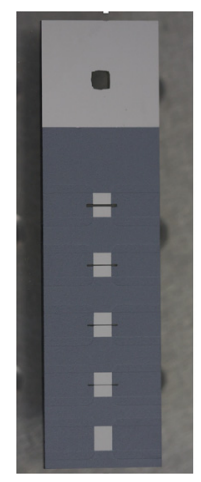

(b)

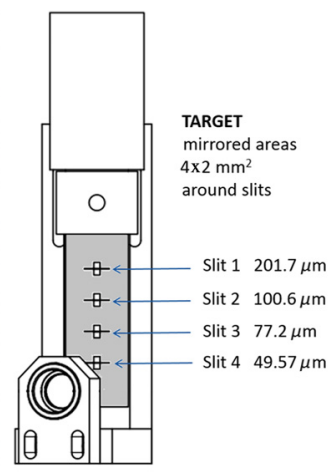

(a)

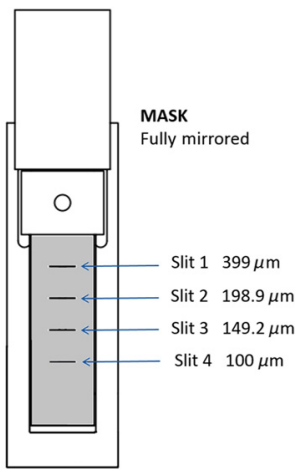

FIG. 5. (a) Image of the target with four slits and a highly reflective surface for OTR: note the selective microsand-blasting treatment that leaves aluminum-coated surfaces close to the slits and on the bottom part for OTR measurements. (b) Final target and mask assemblies as installed on a target holder. The lens holder visible on the target (left) is used for high-magnification imaging of the mirror surface below Slit 4 but is not used in the experiment described here.

(g) Step 7: the photolithography mask for the ODR slits is aligned using the best crystal orientation marks from the previous step, and the photoresist is developed after exposure.

\section{Anisotropic etching of slits in the silicon substrate}

(h) Step 8: another plasma etching is used to clear the $\mathrm{Si}_{3} \mathrm{~N}_{4}$ left without photoresist.

(i) Step 9: the photoresist is stripped.

(j) Step 10: a 10- $\mu \mathrm{m}$ protective layer (ProTEK, shown in yellow) able to withstand $\mathrm{KOH}$ attack is added on the back surface of the wafer to ensure that no punchthrough will happen on the 100-nm layer of $\mathrm{Si}_{3} \mathrm{~N}_{4}$ at the end of the slit-etching process.

(k) Step 11: a 14-h-long $\mathrm{KOH}\left(23 \%\right.$ and $\left.80^{\circ} \mathrm{C}\right)$ etching gets through the $1400-\mu \mathrm{m}$-thick wafer. Since the siliconcrystal orientation is $\langle 100\rangle$, the anisotropic $\mathrm{KOH}$ etching goes 200 times faster in the vertical direction than when following the $\langle 111\rangle$ tilted planes.

(l) Step 12: the protection layer is removed in a mixture of sulfuric acid and hydrogen peroxide (3:1).

(m) Step 13: the $\mathrm{Si}_{3} \mathrm{~N}_{4}$ left is then fully removed by hydrofluoric acid wet etching.

\section{Surface treatment}

(n) Step 14: to enhance the optical reflectivity of the ODR target surface, an 80 -nm aluminum film is evaporated, followed by $30 \mathrm{~nm}$ of $\mathrm{MgF}_{2}$ to protect against oxidation.

(o) Step 15: the wafer is diced into two rectangular ODR targets $\left(20 \times 75 \mathrm{~mm}^{2}\right)$, each one hosting four slits of different sizes separated by $10 \mathrm{~mm}$ (see Fig. 5).

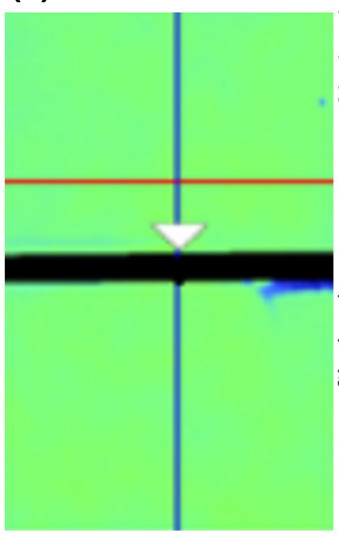

(b)

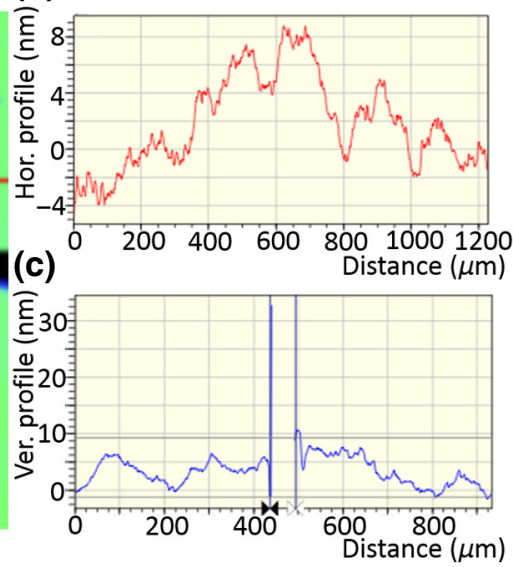

FIG. 6. (a) Detail of a phase shift interference image of a target with a $56-\mu \mathrm{m}$-wide slit (in black). (c) Horizontal and (c) vertical (i.e., across the slit, bottom) surface profiles of the target. The surface flatness is within 8 and $10 \mathrm{~nm}$, respectively.

(p) Step 16: the last step consists in a selective microsand-blasting treatment using a metallic mask to protect the portions where an aluminum coating is required.

The batch of targets produced is characterized using an optical profiler based on phase shift interference (WYCO NT1000) that allows one to measure the 2D surface profile to within a precision of $0.3 \mathrm{~nm}$ over millimeter-scale areas. The measurement of a 56- $\mu \mathrm{m}$ slit presented in Fig. 6 shows that a coplanarity (that is, the flatness across the slit) within $10 \mathrm{~nm}$ is finally achieved. This slit is replaced by a slit with an aperture of $49.7 \mu \mathrm{m}$, shown in Fig. 5, during the experimental campaign. All targets produced are inspected through phase shift interferometry, and only the ones that have surface properties similar to those shown here are actually used in the experiment.

\section{DATA ANALYSIS AND RESULTS}

This section contains the experimental procedure and the acquisition and analysis of the ODR data. We present the procedure as actually performed during a measurement shift at the ATF2 accelerator. After a preparatory phase where the particle beam orbit is optimized and aligned, OTR is used first to measure the beam size at the target location for various accelerator conditions. This allows us to provide a cross-calibration for the actual ODR measurements that are performed under the same beam conditions.

\section{A. Synchrotron-radiation suppression}

SR is the electromagnetic radiation emitted when charged particles are radially accelerated [36]. SR acts as an undesired background in ODR measurements, because 
the SR intensity can be similar to or higher than the ODR signal, resulting in destructive interference and poor reproducibility of the measurements [37,38]. In particular, SR produced upstream and reflected from the internal beampipe surface copropagates quasicollinearly with the beam, so in the angular distribution created in the back focal plane of the lens it is concentrated in the center of the ODR angular pattern, causing an overestimation of the beam size. In particle accelerators, SR is generated not only in dipole magnets but also in quadrupole magnets when the particle beam has an offset with respect to the magnetic center, thus experiencing a dipole deflection. This deflection is routinely used in accelerators for beam-based alignment techniques [39], to align quadrupoles with respect to the beam-orbit center.

Reduction of the SR background is an essential step for an accurate estimation of the transverse beam size, which involves the control of several beam and accelerator parameters such as the orbit and the current in the magnets (and so the strength of the radial acceleration). The insertion of a rectangular mask in front of the target is also essential to achieving the desired SR-background reduction. It has been demonstrated that destructive interference between ODR and SR disappears once the mask is inserted [40]. We describe here the procedure applied before any measurements. The first step of the SR-suppression procedure consists of optimizing the beam orbit using all the steering (corrector) magnets present along the beam line before the instrument to reduce the bending radius locally and to reduce the off-center position of the beam with respect to every quadrupole. The aim is to obtain a "flat" orbit that, after the optimization process, is within $600 \mu \mathrm{m}$ from the field center as measured by the ATF2 BPMs in the vertical and horizontal planes all along the extraction line. The next crucial step is the alignment of the quadrupoles QM14 and QM15, which are the closest elements present before the instrument. This is necessary not only to reduce the SR background [28] but also to a have a stable position of the beam in the target slit when the quadrupole current is varied during scans, as required to measure the beam emittance $[41,42]$. To align the beam with the magnetic centers of the quadrupoles, beam-based alignment is performed, by observing the deflection of the beam center on the target, inserted to produce OTR, through the imaging line. Quadrupoles are mounted on motorized translation stages and are moved transversely until the position of the beam impinging on the OTR target changes when the magnet current is varied. The SR reduction obtained with this technique is clearly shown in Fig. 7 , where the intensity profile of the angular distribution of the vertically polarized OTR is measured before and after orbit optimization. The angular pattern of OTR has a well-known two-lobe structure with a deep minimum in the center [9]. Since SR has a very low angle with respect to the beam propagation direction, its contribution will

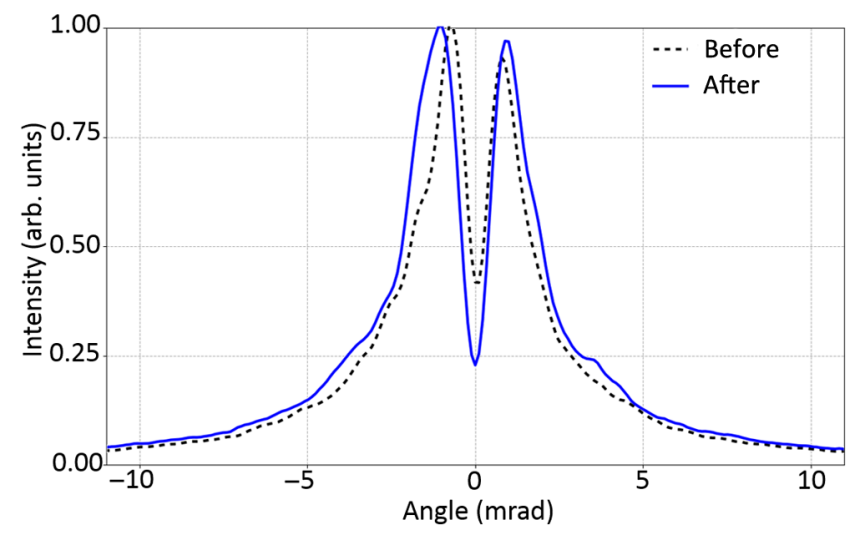

FIG. 7. Intensity profile of angular distribution of vertically polarized OTR measured before and after orbit optimization.

result in an increase in the signal at near-zero angle. It can be seen that, under the same beam conditions at the OTR-target location, the profile after optimization (solid blue line) reaches a level at zero angle that is considerably lower (of the order of 50\%) than the same profile before optimization (dashed black line).

Another important contribution to the reduction of the $\mathrm{SR}$ is the mask present in the instrument that blocks part of the SR generated upstream in the beam line. Just as for the effect of orbit optimization, the presence of the mask was studied making use of the well-known OTR far-field distribution. Figure 8 compares two 2D images of the vertical polarization of the far-field distribution of the OTR recorded by the angular optical line. The presence of a spot in the middle of the pattern (focused SR) is clearly visible without the mask [Fig. 8(a)], which is removed when the mask (in this case a slit of width $399 \mu \mathrm{m}$ ) is inserted [Fig. 8(b)] [28].

\section{B. OTR calibration}

The transverse beam size is obtained from ODR patterns that are calibrated against OTR data under the same (a)

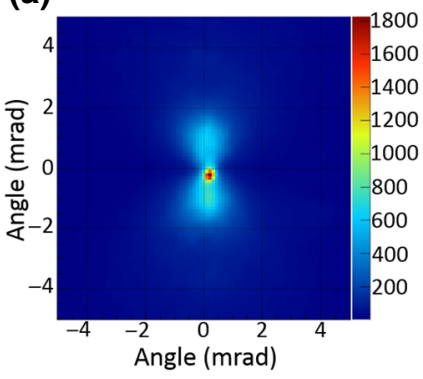

(b)

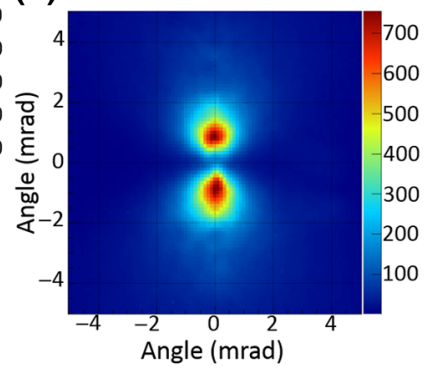

FIG. 8. Angular pattern of vertical polarization of OTR without (a) and with (b) mask. In (a), the SR spot is present in the middle of the two-lobe structure characteristic of the vertical polarization of OTR. 
beam-optics conditions. The reference value provided by OTR is calculated by the procedure presented in Ref. [43]. This allows us to establish a relation between the visibility (which we define in the present section) of the ODR pattern and the transverse beam size. The target is inserted so that the beam intercepts the flat (i.e., without slits) aluminized part of the target. OTR is therefore emitted and observed by the same optical system as that used for the ODR measurements, through the imaging line. Figure 9 shows the intensity of the vertically polarized OTR recorded when the beam is strongly focused vertically by quadrupole QM14, squeezing $\sigma_{y}$. For such a small vertical beam size, the image of the particle beam is dominated by the OTR PSF, showing a two-lobe structure (Figs. 9 and 10). A procedure similar to the one described in Refs. $[43,44]$ is applied to extract the beam size. In particular, an automated quadrupole scan is performed with the QM14 magnet: the current is varied over a large range (from -90 to $-50 \mathrm{~A}$ ), and for each step of the current an image of the OTR is recorded. Then the OTR pattern with minimum visibility is selected, where the visibility is defined as the ratio between the minimum between the lobes and the peak values of the lobes, $I_{\min } / I_{\max }$. Since the intensities of the peaks are often not equal (there is a difference, less than $5 \%$, that can be explained by an optical misalignment of the order of few micrometers), the maximum is calculated as the average value of the two peaks. The OTR profile with minimum visibility is the closest to the expected form for an ideal zero-size particle beam. Then this profile is convoluted with different rms Gaussian profiles that represent real beam sizes, as depicted in Fig. 10. It is possible, in this way, to obtain a calibration curve of the visibility, measured on these convoluted profiles, against the added beam size, as shown in Fig. 11. It can be seen that the curve saturates for small beam sizes of around $1 \mu \mathrm{m}$, showing the limit of the optical system for measuring very small beams.

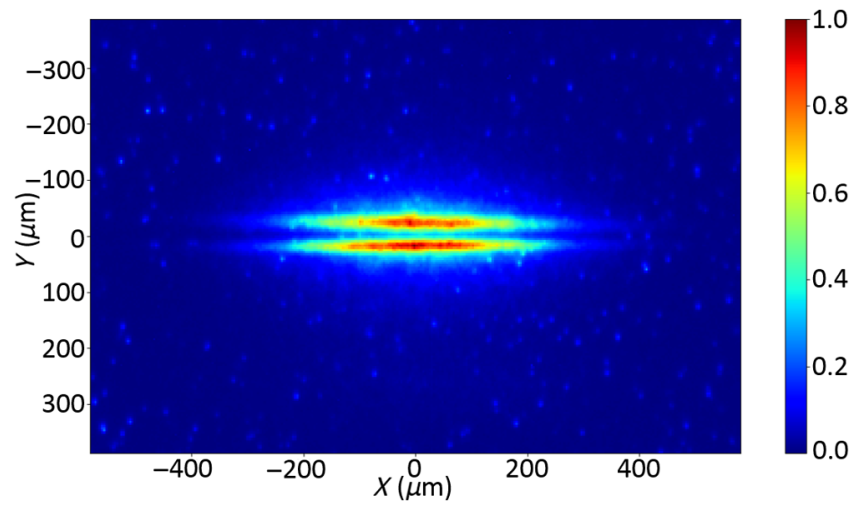

FIG. 9. Vertical polarization of OTR. The beam size is of the order of a few micrometers in the vertical direction $\left(\sigma_{y}\right)$ and a few hundred micrometers in the horizontal direction $\left(\sigma_{x}\right)$. The two-lobe structure is characteristic of OTR emitted by small (i.e., of the order of a micrometer) beams.

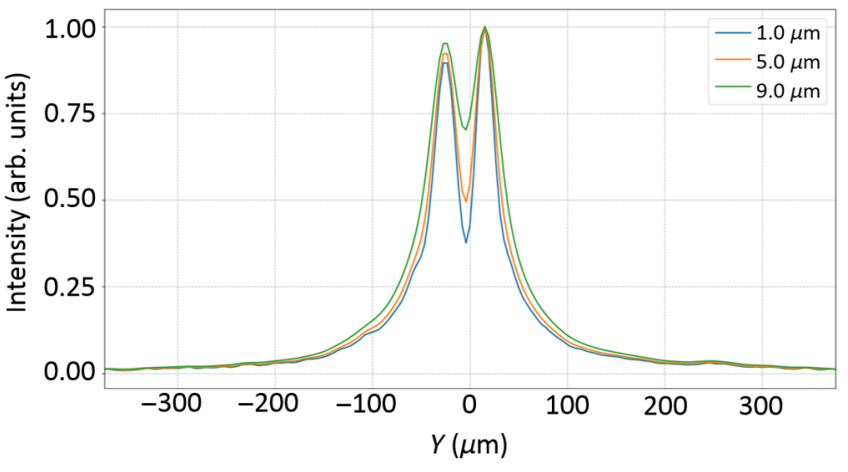

FIG. 10. Profiles of vertically polarized OTR pattern with minimum visibility convoluted with Gaussian curves of $\sigma 1.0 \mu \mathrm{m}$ (blue), $5.0 \mu \mathrm{m}$ (orange), and $9.0 \mu \mathrm{m}$ (green) that simulate the effect of a transverse beam profile on the OTR pattern.

This is the result of a compromise, as the imaging line is on one hand used for OTR calibration, where high resolution (and magnification) is needed, but on the other hand also for monitoring the position of the beam with respect to the slits. In this case a large field of view is needed. A resolution of $1 \mu \mathrm{m}$ is found to be an acceptable compromise. A third-order polynomial is used to fit the curve (orange solid line in Fig. 11). This allows us to associate a beam size with the measured visibility of the OTR during the quadrupole scan.

Figure 12(a) shows the single-shot visibility versus the QM14 current measured from the OTR pattern. The error bars are the rms of the average visibility distribution over 100 images. As can be seen, the errors are bigger closer to a visibility value of 1 , where the two-lobe pattern is almost invisible as it is convoluted with a beam size distribution much larger than the separation between the lobes. This makes the calculation of visibility extremely volatile in this area due to difficult peak fitting. In Fig. 12(b), the beam size versus the quadrupole current is shown, using

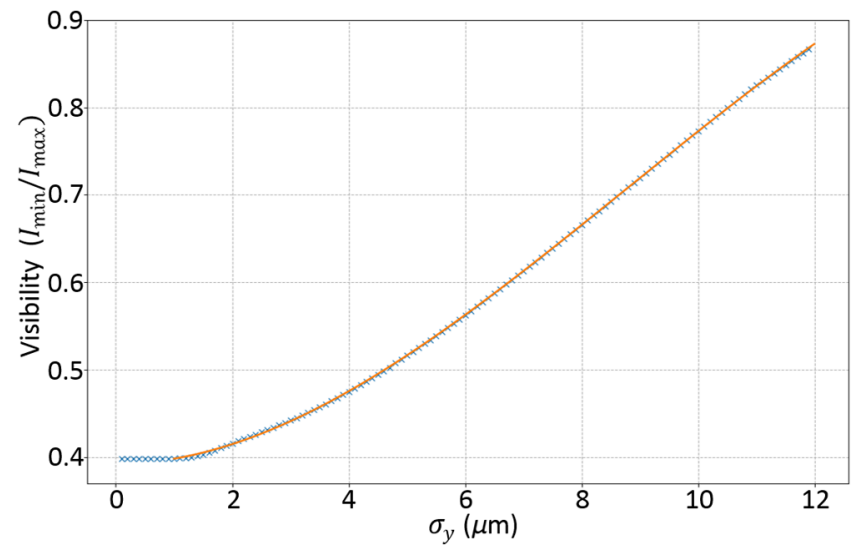

FIG. 11. Visibility of OTR profile as a function of the beam size $\left(\sigma_{y}\right)$. 
(a)

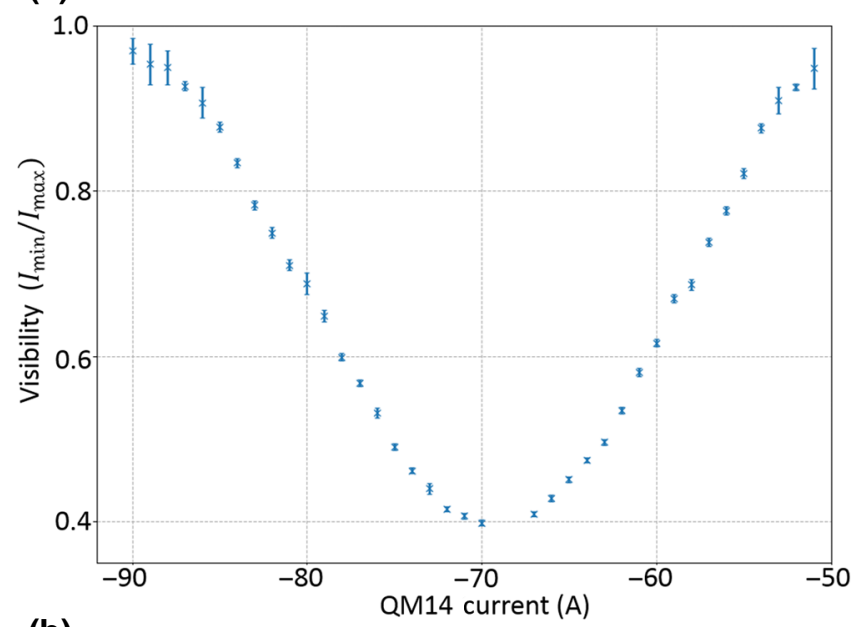

(b)

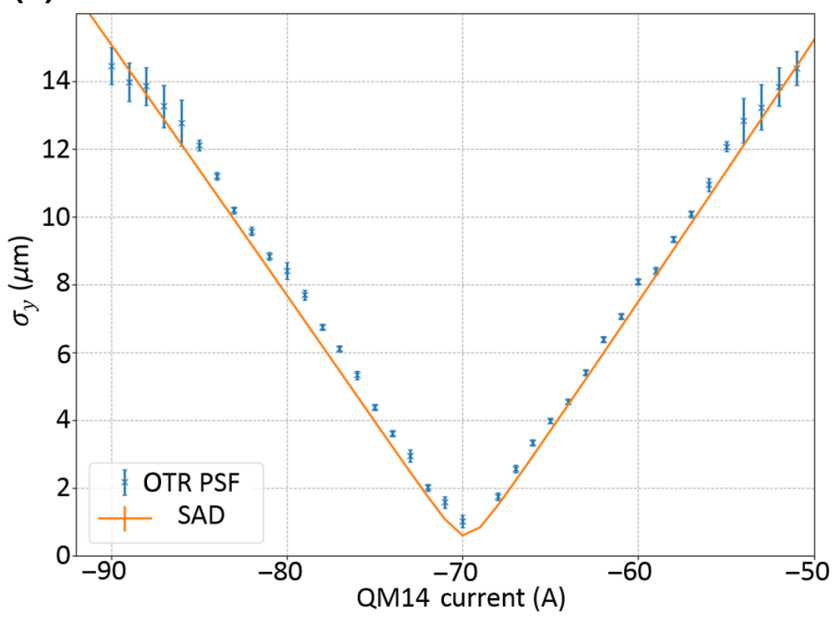

FIG. 12. OTR visibility (a) and measured beam size $\sigma_{y}$, shown as blue points, compared with SAD simulations, shown as a solid orange line (b) for a given range of quadrupole-magnet currents.

the relation found between the visibility values and the beam size (Fig. 11). This allows a relation to be established between the beam size and the current in the QM14 quadrupole for a specific configuration of the accelerator, so that when the ODR angular profile is recorded under the same conditions it can be compared with the expected beam size. We would like to remark that this relation (between beam size and quadrupole current) depends critically on the incoming beam, which depends on the configuration of the accelerator, and the measurement should be repeated every time the machine optics is modified or when the machine is restarted after a shutdown, due to variability of the initial conditions (e.g., magnet hysteresis or photocathode laser fluctuations). The consistency of the OTR cross-calibration is verified by comparing the vertical beam size at the location of the instrument with a strategic accelerator design (SAD) [45] simulation. The beam-optics parameters measured during data acquisition

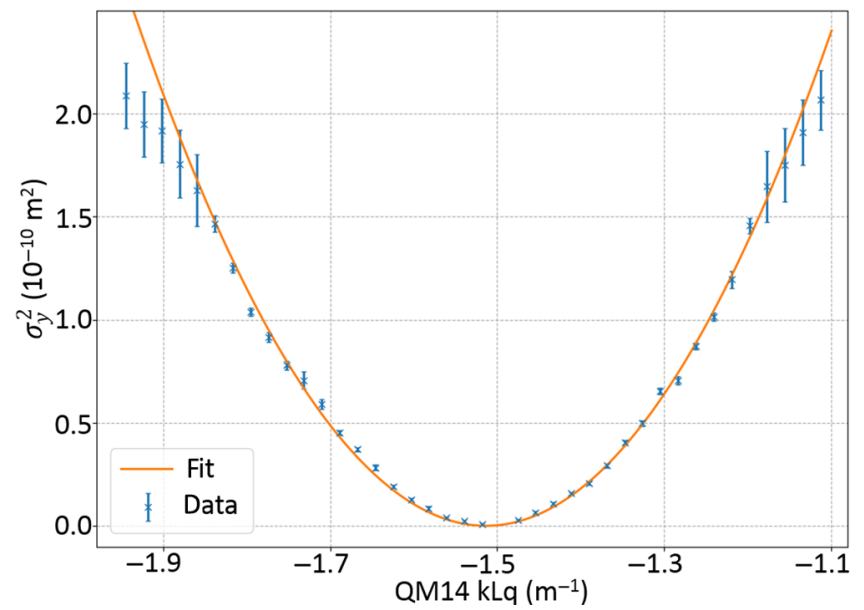

FIG. 13. Parabolic fit (solid orange line) of the square of the beam size measured from the OTR PSF as a function of the quadrupole magnetic strength $\mathrm{kLq}$ to retrieve the beam emittance.

are saved and used as input for the SAD simulation. The results of this comparison are shown in Fig. 12(b) as a solid orange line, in good agreement with the measured beam size.

In particular, the vertical beam emittance considered for the simulation is $34 \mathrm{pm}$. The emittance is also measured from the OTR PSF through a parabolic fit of the beam size squared versus the magnet current, depicted in Fig. 13, using a well-known method described in the literature $[41,42]$.

A second-order polynomial fit

$$
x^{2}=a(1 / f)^{2}-2 a b(1 / f)+\left(c+a b^{2}\right)
$$

is used, where $x^{2}$ is the beam size squared, $1 / f$ is the inverse of the focal length or the quadrupole strength $(\mathrm{kLq})$, and $a, b, c$ are fit parameters. The measured emittance is therefore calculated as $\sqrt{a c} / d^{2}$, where $d$ is the distance between the OTR screen and the quadrupole. From this fit, a value of $33 \pm 3 \mathrm{pm}$ is obtained for the emittance, which is in very good agreement with the simulated beam emittance.

\section{ODR measurements}

The first set of measurements is performed at $400 \mathrm{~nm}$, a wavelength choice that provides a better resolution compared with other longer wavelengths and therefore better sensitivity to small beam sizes, but still in a region of the electromagnetic spectrum of the particles where relatively inexpensive and high-quality optical components are available "off the shelf." In Fig. 14(a), a typical image of the angular pattern of the ODR vertical polarization, measured with a $400-\mathrm{nm}$ filter and a $49.7-\mu \mathrm{m}$-wide slit is shown. The profile integrated between the two red lines is presented in 
(a)

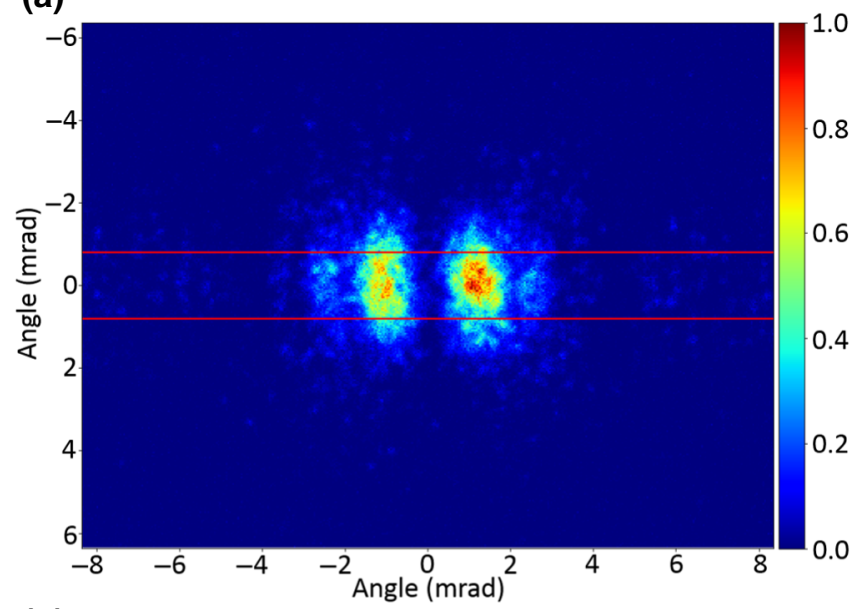

(b)

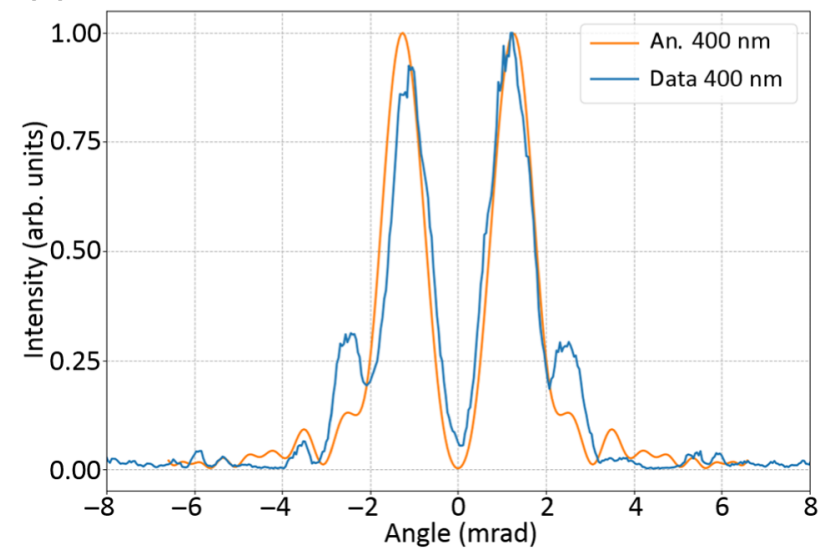

FIG. 14. (a) Vertically polarized ODR at $400 \mathrm{~nm}$ from a 49.57- $\mu \mathrm{m}$-wide slit. (b) The blue line is the profile integrated between the two red lines in Fig. 14(a), and the orange solid line is obtained by an analytical calculation following the model presented in Refs. [18,28].

Fig. 14(b), showing a typical ODR angular pattern [46]. This image is acquired when the beam is strongly focused in the vertical direction by the QM14 quadrupole, leading to a very small visibility of the order of 0.05 . Such small values of visibility could be reached after all of the preparatory steps described in the previous section to reduce as much as possible any stray SR light at very low angles that would have affected the visibility. However, as we will see, a visibility of 0.05 represents the sensitivity limit at 400 $\mathrm{nm}$, as smaller transverse beam sizes do not translate into a variation of visibility.

In an effort to further optimize the sensitivity, measurements in the UV at $250 \mathrm{~nm}$ are also performed. Thanks to careful choice of every component of the optical line (see Sec. IIC), in particular the intensifier in the angular line, with a good sensitivity at wavelengths down to $200 \mathrm{~nm}$, it is also possible to acquire data in the UV at such wavelengths. In Fig. 15(a), an image of the angular (a)

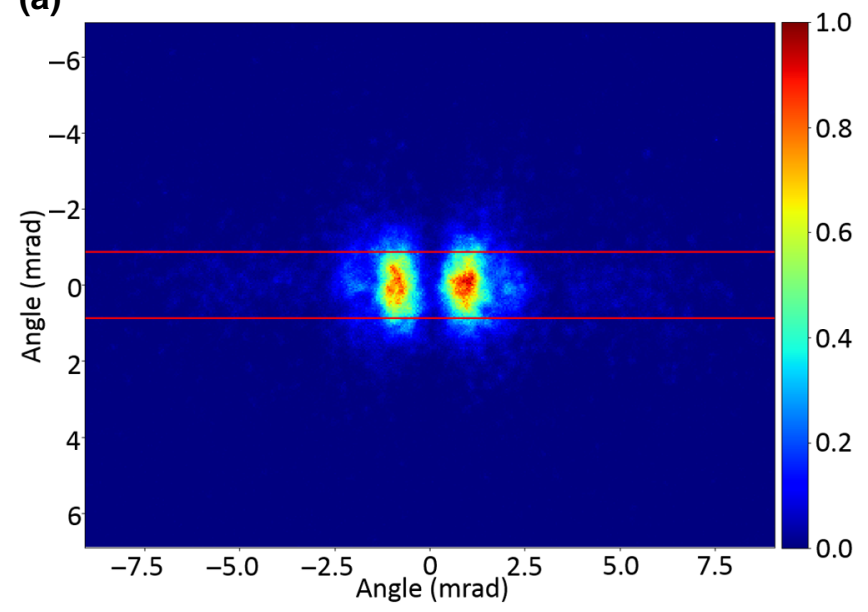

(b)

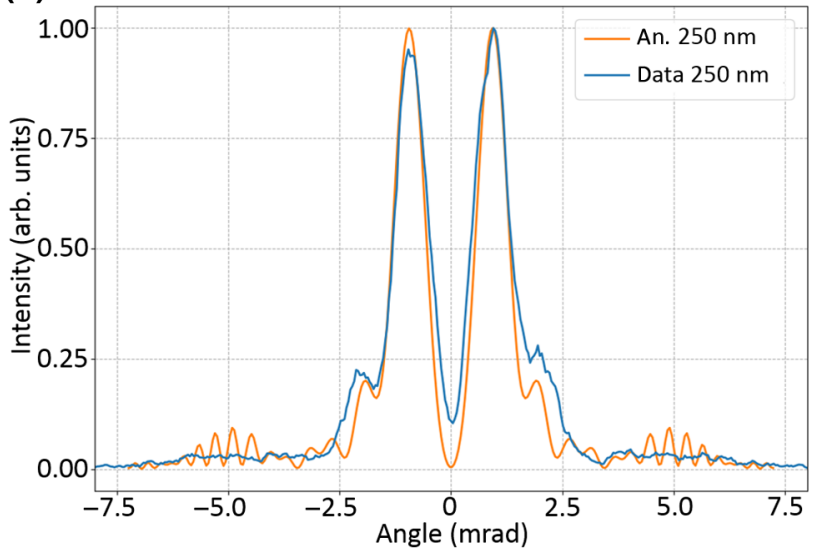

FIG. 15. (a) Vertically polarized ODR at $250 \mathrm{~nm}$ from a $49.57-\mu \mathrm{m}$-wide slit. (b) The blue line is the profile integrated between the two red lines in Fig. 15(a), and the orange solid line is obtained by an analytical calculation following the model presented in Refs. [18,28].

pattern of the vertical polarization of the ODR, saved in the UV, is acquired under the same beam conditions as in Fig. 14(a), showing a slightly smaller ODR pattern with respect to the visible one. The expected contraction of the ODR pattern with respect to the visible-light pattern leads to an increased sensitivity to smaller beam sizes [17]. In this case the visibility, illustrated in Fig. 15(b), shows a larger value than that at $400 \mathrm{~nm}$.

As in the case of the OTR measurements, automated quadrupole scans are performed to record the ODR patterns as a function of the current, i.e., the magnetic strength, of quadrupole QM14. Considering the crosscalibration obtained with OTR explained in the previous section, the current in the magnet can be converted to the expected beam size for a given configuration (beam optics) of the accelerator, allowing us to plot the visibility as a function of the beam size. This is shown in Fig. 16 for the 400-nm (red) and 250-nm cases (blue). It can be seen 


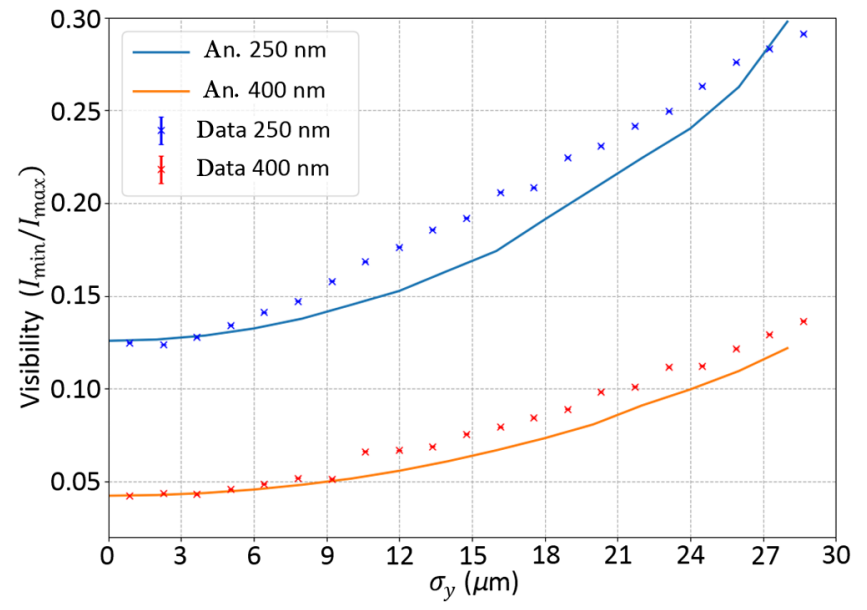

FIG. 16. Visibility as a function of beam size through OTR cross-calibration at $400 \mathrm{~nm}$ (red points) and $250 \mathrm{~nm}$ (blue points). The solid lines are analytical calculations following the model presented in Refs. [18,28].

that the ODR recorded at shorter wavelengths results in an improved sensitivity for small beam sizes, as the variation of visibility is more pronounced and reaches a saturation value at smaller sizes. The solid lines plotted over the two sets of data are analytical calculations following the model presented in Refs. [18,28], which takes into account interference between the target and the mask, and show good agreement in the sensitivity for both wavelengths of observation. The difference for large values of $\sigma_{y}$ can be explained by the fact that the model considers a perfect Gaussian distribution of the beam in the vertical direction, while the real vertical beam profile can be different. The intrinsic limit is around $6 \mu \mathrm{m}$ for $400 \mathrm{~nm}$, while in the $\mathrm{UV}$ at $250 \mathrm{~nm}$ this limit is decreased to about $3 \mu \mathrm{m}$. This represents a substantial improvement of the ODR resolution limit with respect to the previous published result of $14 \mu \mathrm{m}$ obtained at ATF2, although with a different instrument [17]. As during the scan of the QM14 quadrupole current we are varying the vertical beam size at the screen location as the beam waist moves across the target, it is possible to extract the vertical beam emittance from the visibility data acquired, converted to beam size [1]. The data and the fit are presented in Fig. 17 for visible light (400 nm, top) and UV (250 nm, bottom). The measured emittance is $33 \pm 13 \mathrm{pm}$ and $32 \pm 5 \mathrm{pm}$ for 400 and 250 $\mathrm{nm}$, respectively, providing very good agreement with the emittance measured with OTR and simulated with SAD ( $33 \pm 3 \mathrm{pm}$ and $34 \pm 3 \mathrm{pm}$, respectively). The fact that the visible data lead to a larger error in the emittance (i.e., a worse fit shape) compared with the UV measurements is due to the saturation effect at small beam sizes, as can be seen in Fig. 17 for the experimental points between magnetic strengths of -1.8 and $-1.4 \mathrm{~m}^{-1}$.
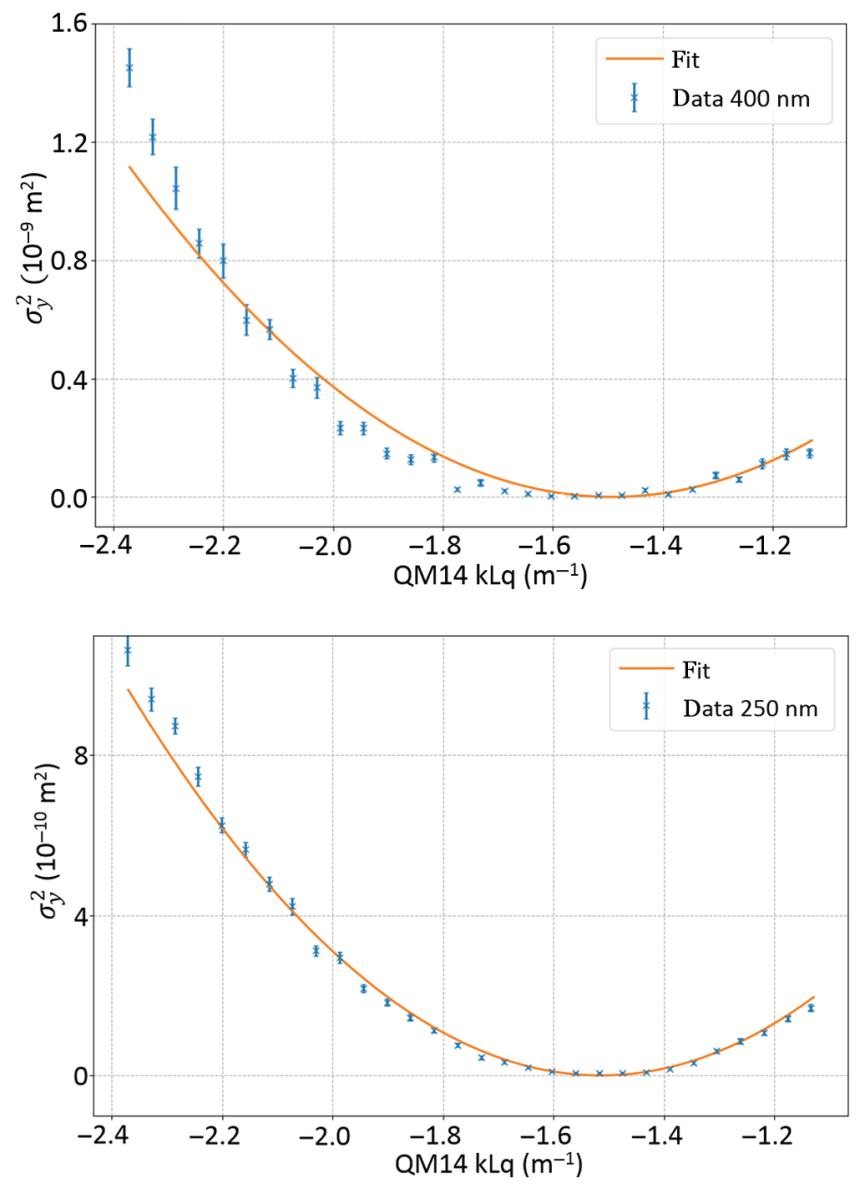

FIG. 17. Square of the beam size measured with ODR for visible light (400 nm, top) and UV (250 nm, bottom) as a function of the quadrupole magnetic strength $\mathrm{kLq}$ to retrieve the beam emittance. A parabolic fit is shown as a solid orange line.

\section{CONCLUSION}

In this paper, we present noninvasive beam size measurements based on ODR in the visible and UV wavelength range, providing clear experimental proof of the longstanding hypothesis that resolution improves when shorter wavelengths are recorded, at the cost of a decrease in radiation intensity. Measurements are performed at the ATF2 beam line at KEK on a $1.3 \mathrm{GeV}$ electron beam with a micrometer-size transverse beam size. An instrument is developed, with a set of micrometer-scale rectangular slits at its core produced by a process that allows stringent surface requirements to be fulfilled to minimize aberrations down to UV wavelengths and to minimize reflection of SR when used in combination with a mask. We develop a procedure for minimizing stray light and other spurious contributions to achieve optimal measurement conditions. When ODR is recorded in the UV at $250 \mathrm{~nm}$, a beam-size sensitivity down to $3 \mu \mathrm{m}$ is demonstrated when crosscalibrated with results from OTR under the same beam conditions. Therefore the system is proven to push down 
the limit of beam size measured with ODR to $3 \mu \mathrm{m}$, much smaller then the previous lower limit of this technique of $14 \mu \mathrm{m}$ presented in the literature [17], to the knowledge of the authors. We define procedures for stray-light minimization and data analysis that make this technique ready for application, although more technical work needs to be performed in order to streamline and automate them as much as possible in a real operational scenario. We consider ODR as a technique that possesses many interesting features, being noninvasive, sensitive to micrometer-size beams, and relatively inexpensive. As we show in the present paper, ODR and OTR detection can be easily combined in a single instrument, where the superior resolution of OTR can be used to cross-calibrate the ODR visibility with the beam size. For potential utilization in future large-scale low-emittance high-intensity linear accelerators, the combined application of these techniques could be the ideal solution for beam size monitoring: OTR for precision measurements in single-bunch mode for machine commissioning, and ODR for continuous monitoring with high-charge bunch trains in multibunch mode during beam operations.

\section{ACKNOWLEDGMENTS}

The authors acknowledge the travel funding provided by the EU through a Europe-Japan Accelerator Development Exchange (E-JADE) grant. The authors would like to express their gratitude to the CLIC collaboration. Thanks must also be given to the ATF collaboration and to all the ATF staff.

[1] S. Y. Lee, Accelerator Physics (World Scientific Publishing Company, Singapore, 2011), 3rd ed., https:/www.worldsci entific.com/doi/pdf/10.1142/8335.

[2] E. J. N. Wilson, An Introduction to Particle Accelerators (Oxford University Press, Oxford, 2006).

[3] H. Hayano, in Linac. Proceedings, 20th International Conference, Linac 2000, Monterey, USA, August 21-25, 2000. Vols. 1, 2, eConf C000821, MOC01 (2000), [146(2000)], arXiv:physics/0008084 [physics.acc-ph].

[4] S. Levasseur, B. Dehning, S. Gibson, H. Sandberg, M. Sapinski, K. Sato, G. Schneider, and J. Storey, Development of a rest gas ionisation profile monitor for the CERN proton synchrotron based on a timepix 3 pixel detector, J. Instrum. 12, C02050 (2017).

[5] P. Forck, in Proceedings, 1st International Particle Accelerator Conference (IPAC'10): Kyoto, Japan, May 23-28, 2010, Conf. Proc. C100523, TUZMH01 (JACoW, Kyoto, 2010).

[6] T. Naito and T. Mitsuhashi, Very small beam-size measurement by a reflective synchrotron radiation interferometer, Phys. Rev. ST Accel. Beams 9, 122802 (2006).

[7] V. Ginzburg and I. M. Frank, Radiation of uniformly moving electron at transition from one media into another one. Zh. Eksp. Teor. Fiz. 16, 15 (1946).
[8] V. Ginzburg, Transition radiation and transition scattering, Phys. Scr. 1982, 182 (1982).

[9] P. Karataev, A. Aryshev, S. Boogert, D. Howell, N. Terunuma, and J. Urakawa, First Observation of the Point Spread Function of Optical Transition Radiation, Phys. Rev. Lett. 107, 174801 (2011).

[10] E. Bravin, T. Lefevre, and C. Vermare, in Proceedings of the Particle Accelerator Conference, 2003. PAC 2003 (IEEE, Portland, 2003), Vol. 4, p. 2464.

[11] L. J. Nevay, S. T. Boogert, P. Karataev, K. Kruchinin, L. Corner, D. F. Howell, R. Walczak, A. Aryshev, J. Urakawa, and N. Terunuma, Laserwire at the accelerator test facility 2 with submicrometer resolution, Phys. Rev. ST Accel. Beams 17, 072802 (2014).

[12] T. Hofmann, K. O. Kruchinin, A. Bosco, S. M. Gibson, F. Roncarolo, G. Boorman, U. Raich, E. Bravin, J. K. Pozimski, A. Letchford, and C. Gabor, Demonstration of a laserwire emittance scanner for hydrogen ion beams at CERN, Phys. Rev. ST Accel. Beams 18, 122801 (2015).

[13] S. T. Boogert, G. A. Blair, G. Boorman, A. Bosco, L. C. Deacon, P. Karataev, A. Aryshev, M. Fukuda, N. Terunuma, J. Urakawa, L. Corner, N. Delerue, B. Foster, D. Howell, M. Newman, R. Senanayake, R. Walczak, and F. Ganaway, Micron-scale laser-wire scanner for the KEK accelerator test facility extraction line, Phys. Rev. ST Accel. Beams 13, 122801 (2010).

[14] M. Castellano, A new non-intercepting beam size diagnostics using diffraction radiation from a slit, Nucl. Instrum. Methods Phys. Res. Sect. A 394, 275 (1997).

[15] M. Aicheler, P. Burrows, M. Draper, T. Garvey, P. Lebrun, K. Peach, N. Phinney, H. Schmickler, D. Schulte, and N. Toge, A Multi-TeV Linear Collider Based on CLIC Technology: CLIC Conceptual Design Report, CERN Yellow Reports: Monographs (CERN, Geneva, 2012).

[16] V. Shpakov and S. Dabagov, Diffraction radiation from an electron beam for one-and two-slit systems, Russ. Phys. J. 55, 1338 (2013).

[17] P. Karataev, S. Araki, R. Hamatsu, H. Hayano, T. Muto, G. Naumenko, A. Potylitsyn, N. Terunuma, and J. Urakawa, Beam-Size Measurement with Optical Diffraction Radiation at KEK Accelerator Test Facility, Phys. Rev. Lett. 93, 244802 (2004).

[18] A. Cianchi, M. Castellano, L. Catani, E. Chiadroni, K. Honkavaara, and G. Kube, Nonintercepting electron beam size monitor using optical diffraction radiation interference, Phys. Rev. ST Accel. Beams 14, 102803 (2011).

[19] A. Cianchi, V. Balandin, M. Castellano, E. Chiadroni, L. Catani, N. Golubeva, K. Honkavaara, G. Kube, and M. Migliorati, First non-intercepting emittance measurement by means of optical diffraction radiation interference, New J. Phys. 16, 113029 (2014).

[20] P. Burrows et al., CLIC Project implementation Plan, Tech. Rep. CERN-2018-010-M (CERN, 2018).

[21] B. I. Grishanov, P. Logachev, F. Podgorny, V. Telnov, D. Angal-Kalinin, R. Appleby, J. Jones, A. Kalinin, O. Napoly, J. Payet et al., Atf2 proposal, vol 2, arXiv:physics/0606194 (2005).

[22] L. Bobb, R. Kieffer, T. Lefevre, S. Mazzoni, T. Aumeyr, P. Karataev, M. Billing, J. Conway, and J. Shanks, Feasibility of diffraction radiation for noninvasive beam diagnostics as 
characterized in a storage ring, Phys. Rev. ST Accel. Beams 21, 032801 (2018).

[23] B. Bolzon, A. Aryshev, T. Aumeyr, S. Boogert, P. Karataev, K. O. Kruchinin, T. Lefevre, S. Mazzoni, L. Nevay, M. Shevelev, N. Terunuma, J. Urakawa, and C. P. Welsch, Very high resolution optical transition radiation imaging system: Comparison between simulation and experiment, Phys. Rev. ST Accel. Beams 18, 082803 (2015).

[24] T. Aumeyr, M. G. Billing, L. M. Bobb, B. Bolzon, E. Bravin, P. Karataev, K. Kruchinin, T. Lefevre, and S. Mazzoni, Advanced simulations of optical transition and diffraction radiation, Phys. Rev. ST Accel. Beams 18, 042801 (2015).

[25] A. Aryshev, G. Blair, S. Boogert, G. Boorman, A. Bosco, L. Corner, L. Deacon, N. Delerue, B. Foster, F. Gannaway, H. Hayano, D. Howell, P. Karataev, L. Nevay, M. Newman, R. Senanayake, N. Terunuma, J. Urakawa, and R. Walczak, Micron size laser-wire system at the ATF extraction line, recent results and ATF-II upgrade, Nucl. Instrum. Methods Phys. Res. Sect. A 623, 564 (2010).

[26] R. Kieffer, M. Bergamaschi, E. Bravin, W. Farabolini, P. Karataev, T. Lefevre, and S. Mazzoni, Experimental Observation of Shadowing in Optical Transition Radiation, Phys. Rev. Lett. 120, 094802 (2018).

[27] P. Karataev, S. Araki, A. Aryshev, G. Naumenko, A. Potylitsyn, N. Terunuma, and J. Urakawa, Experimental observation and investigation of the prewave zone effect in optical diffraction radiation, Phys. Rev. ST Accel. Beams 11, 032804 (2008).

[28] M. Bergamaschi, R. Kieffer, K. Kruchinin, A. Aryshev, N. Terunuma, T. Lefèvre, P. Karataev, and S. Mazzoni, in Proc. IPAC 17, Copenhaghen (JACoW, Copenhagen, 2017).

[29] R. Kieffer, E. Bravin, T. Lefevre, S. Mazzoni, M. Bergamaschi, P. Karataev, K. Kruchinin, M. Billing, J. Conway, J. Shanks et al., Optical diffraction radiation for position monitoring of charged particle beams, Nucl. Instrum. Methods Phys. Res. Sect. B 402, 88 (2017).

[30] Vacuum pump, https:/www.saesgetters.com/sites/default/ files/NEXTORR/20Products/20Data/20sheets_3.pdf.

[31] CCD intensified camera, http://pdf.directindustry.fr/pdf-en/ pco-ag/dicam-pro/29533-93103.html.

[32] Motorized filter wheel, https:/www.owis.eu/en/products/ motorized-positioning-systems/produktgruppe/filterraeder/ produkt/148/.

[33] Image intensifier unit C9547-04, https:/www.hamamatsu. com/eu/en/product/type/C9547-04/index.html.
[34] sCMOS camera, https://www.pco.de/fileadmin/user_upload/ pco-product_sheets/pco.edge_42_lt_data_sheet.pdf.

[35] M. Castellano, E. Chiadroni, and A. Cianchi, Phase control effects in optical diffraction radiation from a slit, Nucl. Instrum. Methods Phys. Res. Sect. A 614, 163 (2010).

[36] A. Hofmann, The Physics of Synchrotron Radiation, Cambridge Monographs on Particle Physics, Nuclear Physics and Cosmology (Cambridge University Press, Cambridge, 2004).

[37] G. A. Naumenko, Synchrotron radiation contributions to optical diffraction radiation measurements, Nucl. Instrum. Methods Phys. Res. Sect. B 201, 184 (2003).

[38] P. Karataev, S. Araki, R. Hamatsu, H. Hayano, T. Muto, G. Naumenko, A. Potylitsyn, N. Terunuma, and J. Urakawa, Observation of optical diffraction radiation from a slit target at KEK accelerator test facility, Nucl. Instrum. Methods Phys. Res. Sect. B 227, 158 (2005), Radiation from Relativistic Electrons in Periodic Structures (RREPS'03).

[39] P. Tenenbaum and T. Raubenheimer, Resolution and systematic limitations in beam-based alignment, Phys. Rev. ST Accel. Beams 3, 052801 (2000).

[40] P. Karataev, R. Hamatsu, H. Hayano, T. Muto, N. Terunuma, J. Urakawa, A. Naumenko, and A. Potylitsyn, in Proceedings of European Particle Accelerator Conference EPAC04, EPAC 04 (Lucerne) (JACoW, Lucerne, 2004), Vol. 2, p. S1.

[41] S. Bernal and D. Stratakis, Emittance Measurement: Quadrupole Scan (U.S. Particle Accelerator School 2008, U. of Maryland, College Park, 2008).

[42] A. Green and Y.-M. Shin, in Proc. IPAC 15, Richmond (JACoW, Richmond, 2015).

[43] K. Kruchinin, A. Aryshev, P. Karataev, B. Bolzon, T. Lefevre, S. Mazzoni, M. Shevelev, S. T. Boogert, L. J. Nevay, N. Terunuma, and J. Urakawa, Sub-micrometer transverse beam size diagnostics using optical transition radiation, J. Phys. Conf. Ser. 517, 012011 (2014).

[44] A. Aryshev, S. Boogert, D. Howell, P. Karataev, N. Terunuma, and J. Urakawa, A novel method for submicrometer transverse electron beam size measurements using optical transition radiation, J. Phys. Conf. Ser. 236, 012008 (2010).

[45] SAD, Strategic Accelerator Design, is a computer program for accelerator design, see http://acc-physics.kek.jp/SAD.

[46] R. Fiorito and D. Rule, Diffraction radiation diagnostics for moderate to high energy charged particle beams, Nucl. Instrum. Methods Phys. Res. Sect. B 173, 67 (2001). 